\title{
In-flight analysis of intracranial pressure in pilots undergoing variation in $\mathrm{Gz}$
}

\begin{abstract}
Intracranial pressure (ICP) is an important clinical variable that remains inaccessible to doctors and aerospace professionals. Ten Pilots of the Brazilian Air Force Academy (AFA) were monitored with a Brain care Non-Invasive Intracranial Pressure Sensor during looping maneuvers. The intracranial pressure sensor used was the model BcSs-1000, developed by the Brain care team. The pilot performed the flight in the T-25 Aircraft used in the aviator training course at the Brazilian Air Force Academy (AFA).The ICP of the volunteers was monitored on the occipital bone using a sensor attached to the pilots' helmets. The ICP measuring device was attached to a bag and fixed to the pilot. The monitoring was carried out during a 30minute flight. Pilots were instructed to perform one acrobatic looping with an average duration of 30 seconds. The stunts were performed in the fifteen minutes of the flight. The maximum $\mathrm{Gz}$ load of these acrobatic maneuvers was measured as $+3 \mathrm{Gz}$. It was observed in the results that during the execution of the looping acrobatics, there was an increase in the values of area, height and amplitude of the ICP peaks. The results showed that there was an increase of approximately $46.25 \%$ of the peak of ICP after the beginning of the looping. When compared to the ICP during the looping, the values found were $55 \%$ lower. By observing all the results, human exposure to Load Gz raises the values of the ICP and can have the consequence of an increase in arterial pressure.
\end{abstract}

Keywords: intracranial pressure, acrobatic maneuvers, cardiovascular alterations, loss of consciousness
Volume 2 Issue 3 - 2018

\author{
Thiago Augusto Rochetti Bezerra, \\ Deusdedit Lineu Spavieri Júnior, ${ }^{2}$ Gustavo \\ Frigieri, ${ }^{2}$ Rodrigo Brunell, ${ }^{2}$ Sérgio \\ Mascarenhas de Oliveira ${ }^{1,2}$ \\ 'São Carlos Institute of Physics, Brazil \\ ${ }^{2}$ Braincare Health Technology, Brazil
}

Correspondence: Thiago Augusto Rochetti Bezerra, São Carlos Institute of Physics, São Paulo, Brazil,

Email thiago_rochetti@globomail.com

Received: October 23, 2017 | Published: May 102018

\section{Introduction}

Flight fatigue is defined as a state determined by aerial activity that deteriorates the psycho physiological condition of the crew member, causing a progressive decrease in their performance. ${ }^{1-3}$ Situations that generate flight fatigue can be grouped as follows:

a. Those from the air activity itself, called operational factors,

b. Pilot physiological factors. ${ }^{4-6}$

These professionals can be submitted to extreme physiological conditions, and, consequently, lack of physical preparation or clear perception of some symptoms can lead to physical failure, even death. ${ }^{7.8}$ Although technological developments have provided advances in ergonomic designs, software, hardware, and air traffic control techniques-which has had a positive impact on flight safety-the presence of the human factor remains a prominent cause of aeronautical accidents. ${ }^{9-11}$ Space disorientation is a significant factor in a large percentage of military aviation incidents. While previous studies have analyzed accident statistics, they often suffer from methodological flaws, leading to questionable conclusions about the true causes of accidents with civilian and military aircraft. ${ }^{12,13}$ Acrobatic flight can significantly alter a pilot's ability to orient themselves spatially. In this way, factors inherent to the human physiology associated with aerial activity should be investigated; intracranial pressure (ICP) is an important clinical variable that remains inaccessible to doctors and aerospace professionals. ICP is the pressure inside the cranial cavity. Three components fill this space: blood, cerebrospinal fluid, and brain tissue, and alterations in one or more of these components leads to variations in intracranial pressure ${ }^{14}$ such as oscillations in arterial blood pressure.
Exposure to high $\mathrm{Gz}$ acceleration can lead to changes in blood pressure and redistribution of blood volume. The Gz load is classified into two types: the Gz load is positive when the aircraft moves in the opposite direction to the component of the gravity force and acceleration is greater than that of gravity $\left(9.8 \mathrm{~m} / \mathrm{s}^{2}\right)$; and the $\mathrm{Gz}$ load is negative when the aircraft is in inverted flight (back flight), or its direction is the same as the gravity force, but with acceleration greater than gravity. The cardiovascular alterations in positive or negative Gz result from an increase in the hydrostatic gradient present in the venous and arterial systems. ${ }^{15,16}$ Positive Gz increases cerebral blood flow, while negative $\mathrm{Gz}$ directs blood flow to the lower limbs. The high positive Gz forces found in tactical military aviation and acrobatic flights produce a series of physiological responses designed to preserve cerebral perfusion. Some air forces have instituted measures through anti-Gz suits to increase the physiological response in order to avoid G-induced (G-LOC) loss of consciousness and the potentiality to cause a catastrophic accident. ${ }^{17}$ High GZ acceleration effects can threaten flight safety through loss of consciousness or a lesser known phenomenon, G-induced vestibular dysfunction (GIVD). There are reports of GIVD after high-speed exposure or exposure to the centrifuge. ${ }^{13,18}$ Exposure to high accelerating $+\mathrm{Gz}$ forces in a centrifuge or aircraft can severely decrease cerebral blood perfusion and cause rapid G-induced loss of consciousness. ${ }^{1,18}$ However, smoother acceleration can gradually reduce cerebral blood flow and affect cognitive function. ${ }^{1}$ This study used intracranial pressure monitored noninvasively to understand the effects of $\mathrm{Gz}$ alterations on cerebral hemodynamic and intracranial pressure.

\section{Materials and methods}

Ten Pilots of the Brazilian Air Force Academy (AFA) were 
monitored with a Brain care Non-Invasive Intracranial Pressure Sensor during looping maneuvers. The pilots performed the flight in a T-25 Aircraft used in the aviator training course at the Brazilian Air Force Academy (AFA). The ICP of the volunteers was monitored on the occipital bone using a sensor attached to the pilots' helmets. A Brain care Monitor BcMM-2000 (Brain care Health Technology) was used to monitor and record data with a sampling rate of $200 \mathrm{~Hz}$. The intracranial pressure sensor used was the model BcSs-1000, developed by the Brain care team. The ICP sensor was attached to a bag and fixed to the pilot. The monitoring was carried out during a 30 minute flight. Pilots were instructed to perform one acrobatics Loopings with an average duration of 30 seconds. The stunts were performed in the fifteenth minutes of the flight (Figure1). The maximum Gz load of these acrobatic maneuvers was measured as $+3 \mathrm{Gz}$. Data were collected in an SD card and analyzed off line.

\section{Data analysis}

The data analysis is composed by following processes:

a. Artifact identification;

b. signal decomposition into trend and Pulsatile components;

c. Pulse identification, separation and alignment;

d. Statistical analysis;

We first separate the artifact based on the windowed power spectral density ratio (SNR) between of the pulsatile component-frequencies between 0.5 and $3 \mathrm{~Hz}$ and raw signal up to $40 \mathrm{~Hz}$. We decomposed the signal into trend and Pulsatile components by subtracting the DC component of the low pass signal (FIR filter with cutoff frequency of $20 \mathrm{~Hz}) .{ }^{19}$ From the Pulsatile component, we identify each pulse using the phase of the Hilbert transform. ${ }^{20}$ We perform a second artifact elimination by identifying pulses that had amplitude or length $65 \%$ above or below the averages pulse properties. After pulse separation, we aligned iteratively all valid pulses using linear correlation between each pulse and mean pulse of period. We calculated the mean pulse and corresponding confidence interval by non-parametric bootstrap with $\alpha=0.05$ e one thousand replications..$^{21}$

We calculate the mean pulse properties - height, area, peaks positions, time to peak, length and inflection points-by local search of numerical maxima and minima on pulse waveform and its derivatives. Statistical inference on pulse properties was done using nonparametric statistical tests. ${ }^{22}$ All safety standards were adhered to in the study. All subjects were experienced with exposure to high $\mathrm{Gz}^{+}$. The National Ethics Committee approved the project (CAAE: 40667114.7.0000.5504), and financial support was received from the São Paulo Research Foundation (FAPESP-Process no. 2014/218037).

\section{Results and discussion}

The results are presented in figures. The total area of the curve, the height and amplitude of the ICP peak, and the total time of the pulses in seconds were measured. Blood volume mean per cardiac pulse was indirectly estimated. The height of the peak determined the maximum variation in blood volume during each heartbeat. It is known that total peak time is inversely proportional to the resistance of the cerebral vascular system, i.e., the longer the total pulse time, the lower the cerebral vascular resistance. ${ }^{23}$ It was observed in the results that during the execution of the looping acrobatics, there was an increase in the area, height, and amplitude values of the ICP peaks (Figure 2). It was observed that in $70 \%$ of the samples a decrease in the IPC pulses occurred after the looping when compared to the same values before the looping (Figure 3). The total decrease in the area was small, only $1.71 \%$. The heart rate of the pilots decreased $1.67 \%$ in this comparison. It is important to highlight in the above results that in all cases there was an increase in ICP during looping. In order to discard the hypothesis that this increase was due to the movement of the pilot's head during the looping, we performed tests that verified that the change in head direction did not influence the results of the increase in ICP.

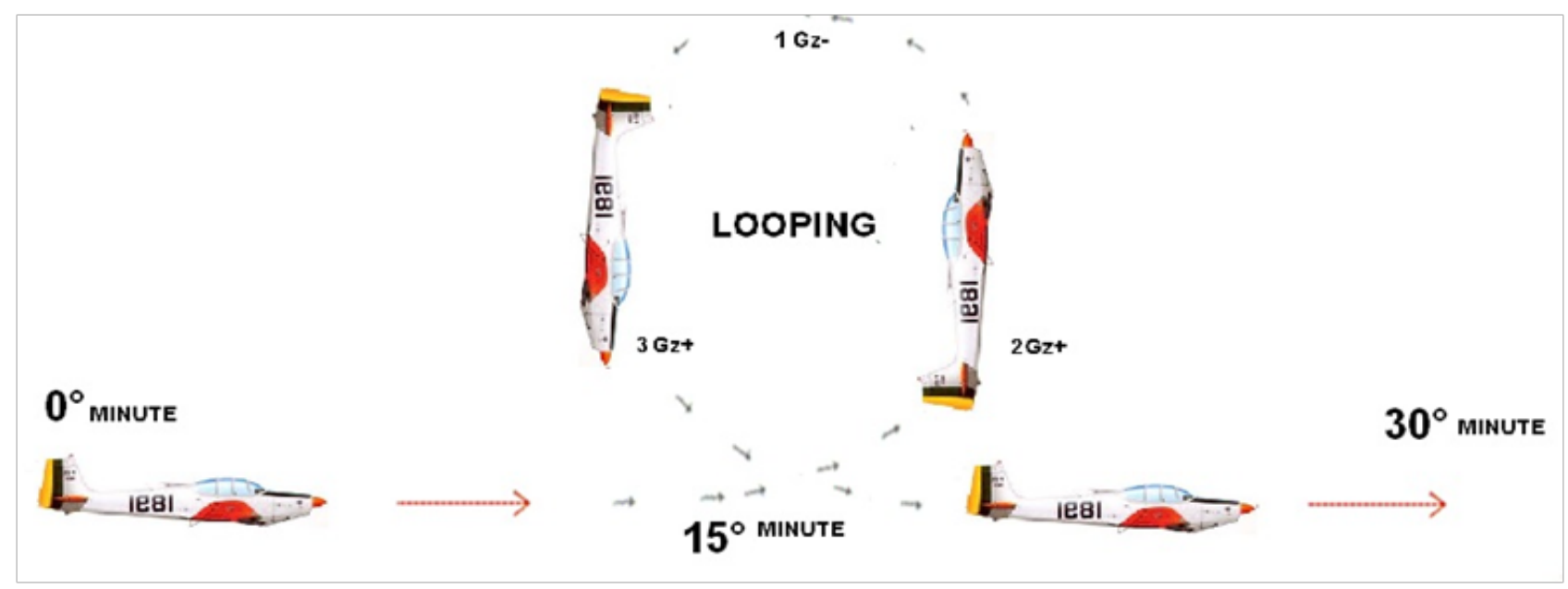

Figure I Monitoring ICP during looping. 


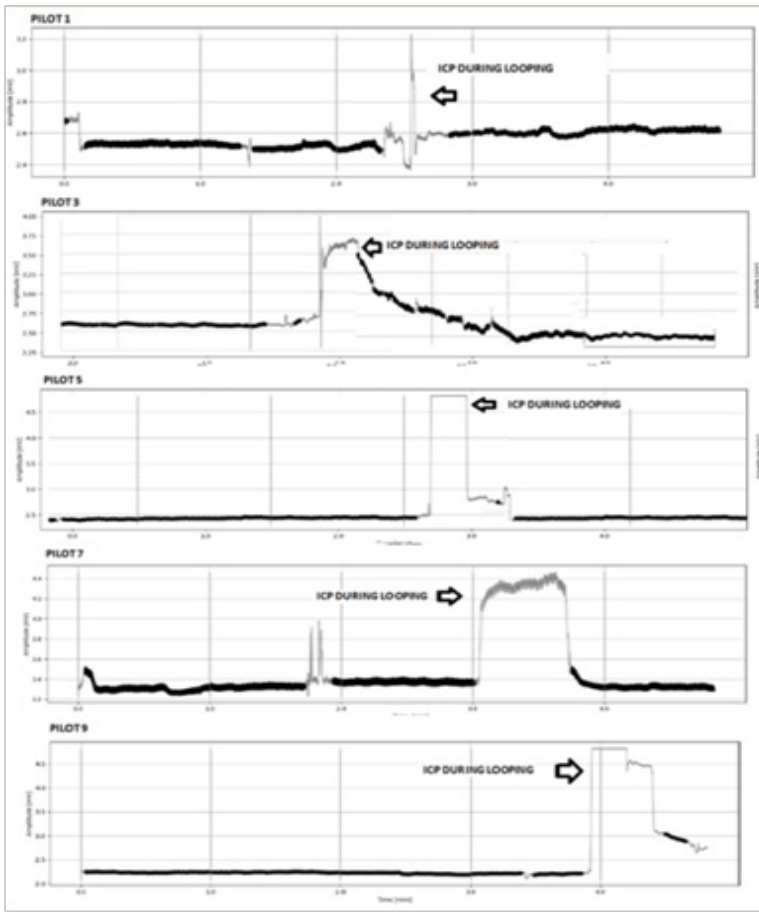

Figure 2 Measurement of area of pilots during ICP looping.

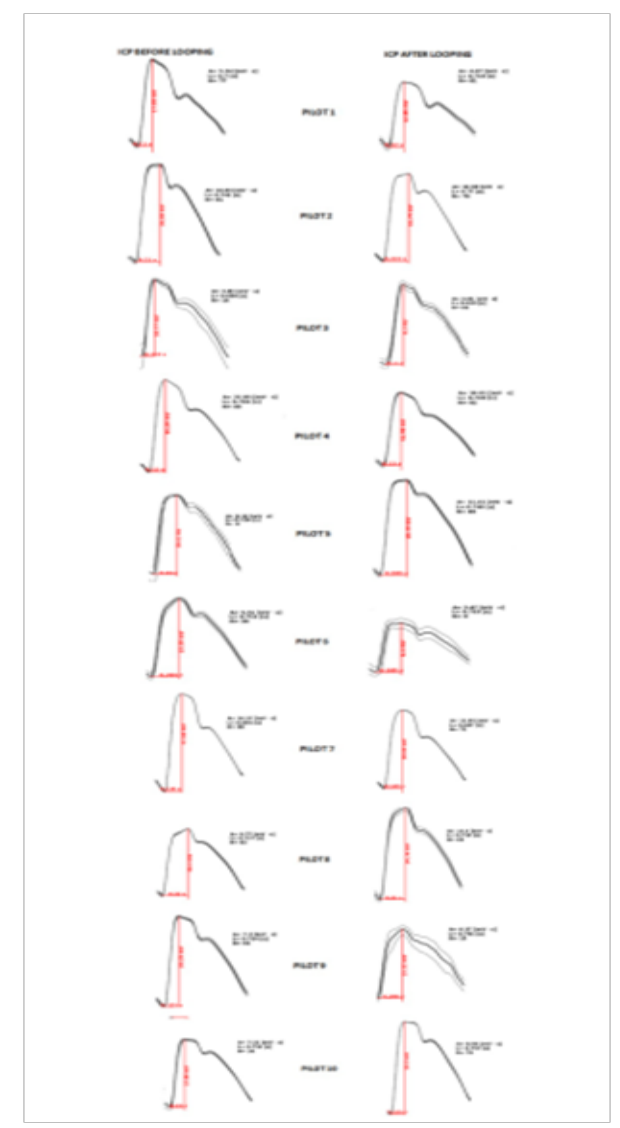

Figure 3 The ICP before and after looping.

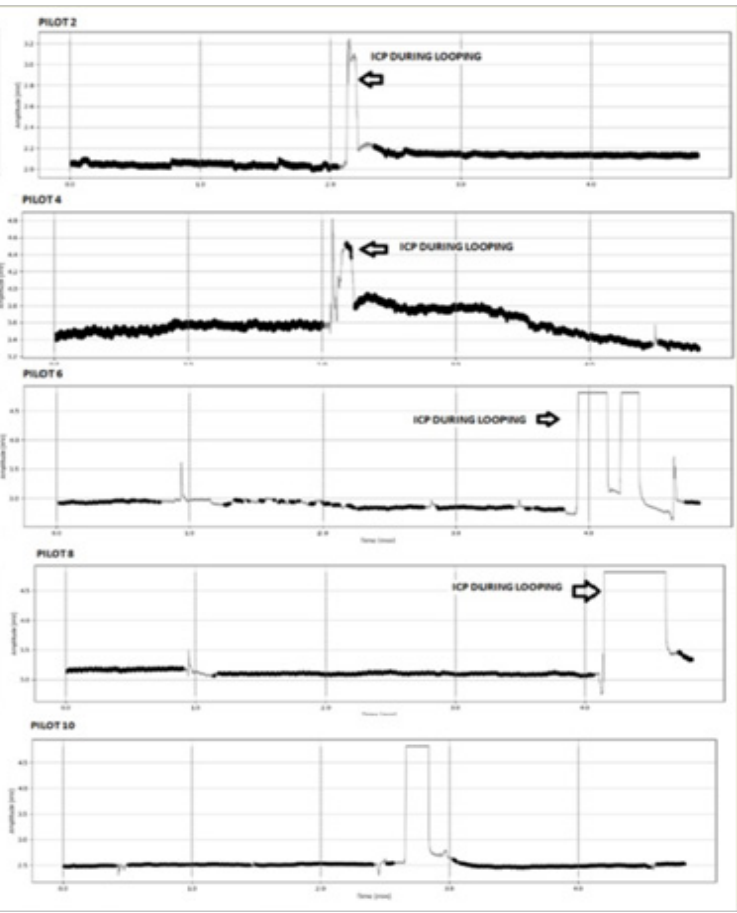

Although this is a broadly speculated theme, largely theoretical, intracranial hypertension induced by flight with $\mathrm{Gz}$ gravitational variation has gained acceptance as a distinct clinical phenomenon, yet the underlying physiological mechanisms are still poorly understood. Symptoms of malaise, nausea, vomiting, vertigo, headaches, and visual pathologies and disturbance are commonly associated with an increase in ICP, both on land and in flight. ${ }^{27,28}$ The most likely mechanisms of ICP include cephalic displacement of body fluids, obstruction of venous flow, largely induced by increased $\mathrm{Gz}$, and which may result in increased Arterial and Intracranial Pressure. ${ }^{30,31}$ The great difficulty for researchers is the realization of an experiment in flight. The major Aerospace Medicine centers carry out experiments with variation in Gz load in human centrifuges. The present study, however, sought new information in flight associating non-invasive equipment to measure the ICP, with variations in the Gz load., ${ }^{9,30,31}$ The difficulties in carrying out this experiment were great, among them adapting the sensor to the helmet and carrying out the collections in flight. Another difficulty was recording the data without electromagnetic influence of the aircraft, all this in association with flight safety of the evaluated pilot. The partnership between the researchers and the Brazilian Air Force was fundamental to the success of the project. In this way, a considerable amount of time was spent so that the collections would present real data and with credibility to our research. Long-term exposure to an increase or absence of gravity has aroused the concern of the aerospace medical community as its effects on the nervous system are unknown. ${ }^{32}$ One of the objectives of this experiment was to establish whether there is an increase in blood flow to the brain and as a consequence an increase in ICP during human exposure to an increase in Gz. There is evidence in the results of this work that corroborate with this objective.

In the short term the symptoms of increased ICP are associated with momentary loss of vision due to G-LOC. A new emerging science 
in human factors known as neuroergonomics seeks to understand human performance in complex systems, in relation to underlying brain mechanisms, and to use this knowledge to improve the human in-flight performance system..$^{32}$ This research area deals with a major threat for modern fighter jet pilots known as gravity-induced loss of consciousness, which occurs during sudden surges or acrobatics. ${ }^{7}$ The G-LOC is caused by a sudden reduction in brain $\mathrm{O}_{2}\left(\mathrm{rSO}_{2}\right)$ as a result of the increase in the + Gz force. ${ }^{2,33}$ From 1982 to 2002, G-LOC was responsible for the loss of 24 US Air Force pilots and several nonfatal accidents. ${ }^{13,15,34}$ When the excessive Gz force is removed and the aircraft returns to level flight, $24 \mathrm{~s}$ of functional impoverishment are expended. ${ }^{35,36}$ The study indicated, however, that the course of the G-LOC episode is much longer than initially believed. These data corroborate with ours. In the present experiment it was observed that in $70 \%$ of the samples there was a reduction in the ICP pulses after the looping when compared to the same values before the looping, however it took a few minutes for these values to be normalized. This time may be sufficient to cause an accident if G-LOC occurs or there is an exponential increase in ICP. Given the capability of the Modern Hunt aircraft, a pilot with G-LOC symptoms at a speed of 500mph can fly approximately 12 miles while not having control of the aircraft. ${ }^{2}$ What may explain the prolonged delay of seconds or minutes in the recovery of flight and emergency performance is the state of relative disability. One possibility is that $\mathrm{rSO}_{2}$ returns very slowly after the gravitational force produces the G-LOC. This possibility is suggested by the fact that during $\mathrm{Gz}$ acceleration blood is concentrated in the abdominal cavity and lower limbs and may therefore require time to return to normal cortical circulation. ${ }^{33,37}$ In addition, other studies have shown that there is a decrease in $\mathrm{rSO}_{2}$ caused by a decrease in the oxygen level of the air they are breathing (hypoxic), and recovery of $\mathrm{rSO}_{2}$ when atmospheric oxygen is restored to a normal level is slow, approximately 90 seconds. Although the reductions in $\mathrm{rSO}_{2}$ during $\mathrm{Gz}$ acceleration have been well documented, it is important to note that few data are available about the in flight rate of return or increase in ICP. ${ }^{9,23,25,38}$

Another concern is in the absence of gravity. The majority of astronauts need only 2 to 3 days to acclimate to microgravity. The more time spent in space, the more overlapping physiologically distinct symptoms appear to arise, including headaches and visual disturbances. These findings are similar to cases of intracranial hypertension in the terrestrial environment caused by elevated ICP. ${ }^{25}$ Long-term exposure to microgravity has aroused the concern of the aerospace medical community as its effects on the nervous system are unknown. The authors determined that these findings were representative of intracranial hypertension through elevated ICP. ${ }^{25}$

We sought in this work to initiate a study that relates the ICP with elevated exposure to an increase in $\mathrm{Gz}$, since a pilot of the Brazilian Air Force has between 3000 and 5000 hours of flight during their career, a good part of this being exposed to variation in gravity. Clinically, visual pathology is considered a measure sensitive to ICP, as the perineural subarachnoid space of the optic nerve is contiguous with the intracranial space and therefore vulnerable to fluctuations in ICP. The increase in ICP places a short-term risk on the operational mission, contributing to the long-term risk that has not yet been fully elicited. Individuals with idiopathic intracranial hypertension (iih) are documented with severe headaches and loss of vision, but may also experience ataxia, memory disorders, and cognitive dysfunction. Several studies have revealed deficits in patients with ICP, especially in memory tests, reaction time tests, speed processing, visuospatial memory, and attention compared to a demographically paired healthy control group..$^{23}$ This condition may serve as a predictor for future flight sequelae in aeronauts. Intracranial hypertension could also represent a commercial problem. The incidence of intracranial hypertension may rise with the increase in civilian space travelers who are not as physiologically skilled as pilots. In addition, increased ICP in the environment may become more concerning in someone who has a predilection, or underlying disease process that, combined with increased ICP, can cause flight or post-flight problems. ${ }^{25}$

Only one of the samples showed an perfect increase in PCI as a result of Looping. In all other samples, after the peak increase, there was signal saturation. To avoid signal saturation, adjustments must be made to the equipment in future studies. The Figure 4 presents the difference between amplitude in this sample, area, and peak height before, during, and after looping. The results demonstrate that there was an increase in the peak ICP after the beginning of the looping of approximately $46.25 \%$. When compared to the ICP during the looping, the values found were 55\% lower. Observing all the results, it is demonstrated in Figure 4 that human exposure to a change in $\mathrm{Gz}$ load raises the values of the ICP and can result in an increase in the AP. When ICP increases, the venous pressure increases concomitantly and, consequently, the damping effect of the veins is reduced, resulting in an increase in blood pressure. ${ }^{24}$ The Figure 5 demonstrates that in one of the samples there was an increase in the peak of $24.4 \%$ of ICP during looping. This figure shows that there was an increase in the amplitude of the ICP pulses due to the increased Gz load during performance of the looping. It can be seen in Figure 5 that there was an increase in the amplitude of the ICP pulses during the looping. The result suggests that raising the LCR pulse amplitude (pulse pressure) increases with the ICP according to the exponential form of the craniospinal volume-pressure curve. The magnitude of pulse pressure is determined by the shape of the curve and by the quantity of Pulsatile alteration in cerebral blood volume. ${ }^{25}$ Figure 6 shows the CPI pulse, amplitude, and time to normalize the pulse. With respect to the increase in amplitude, the literature reveals that cerebral blood flow is not completely interrupted during the $\mathrm{Gz}^{+}$increase, but may decrease by up to $50 \%$. This depends on the amount of Gz to which the pilot is exposed and the duration of this exposure. Cerebral oxygenation decreases if acceleration is maintained (cerebral hypoxemia), with a decrease in oxygen saturation in arterial and venous blood. ${ }^{18,24,26,27}$

\section{Conclusion}

Variation in intracranial pressure is a very common event, and may even occur in the case of simple encephalitis, or severe cases that require deep and appropriate knowledge for monitoring and subsequent treatment. It is necessary that the medical profession at least dominate and understand ICP. The techniques described in this work to obtain an intracranial pressure signal in flight required knowledge from the evaluators.

Currently the techniques for non-invasive ICP monitoring are still under study. From the moment the signal is obtained, it is possible to draw conclusions about other vital parameters such as heart and respiratory rate, through adequate filtration and processing of the 
signal received. Thus, it is possible to save time and resources that may be essential in diagnosis. For future studies we suggest the analysis of blood pressure continuously together with ICP (Prx), and measurement of $\mathrm{rSO}_{2}$. Our team of researchers is already developing equipment that performs the monitoring of multiple parameters simultaneously, allowing reaction to a crisis situation and the creation of a strategy for the care of the evaluated individual. This study verified that an increase in ICP occurs due to acrobatic flight with variation in $\mathrm{Gz}$. The literature is still scarce on the problem of cranial hypertension in the real space flight environment, and further studies are needed if this phenomenon is to be properly understood.

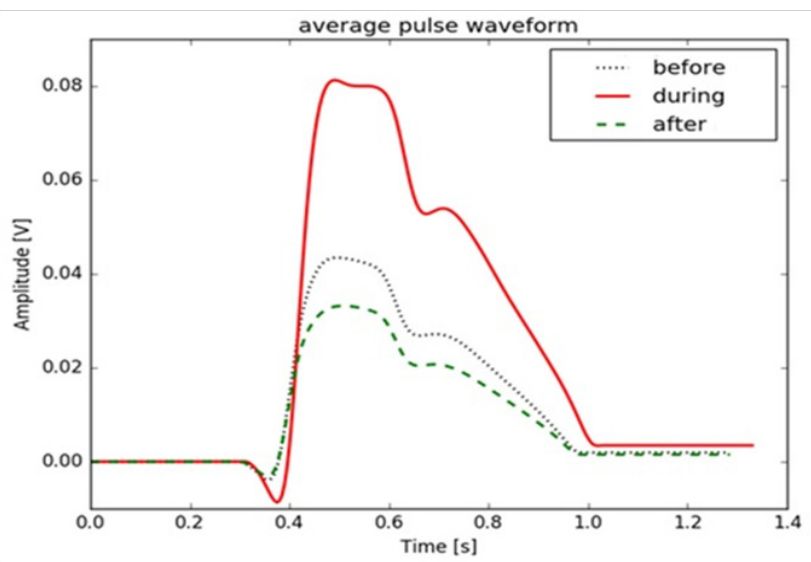

Figure 4 Average pulse waveform before, during and after the looping.

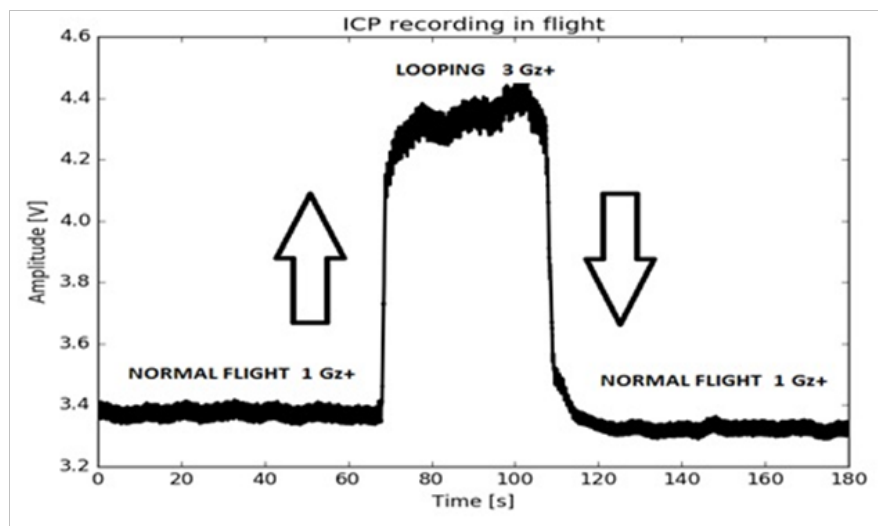

Figure $5 \mathrm{ICP}$ recording in flight in pilot 7.
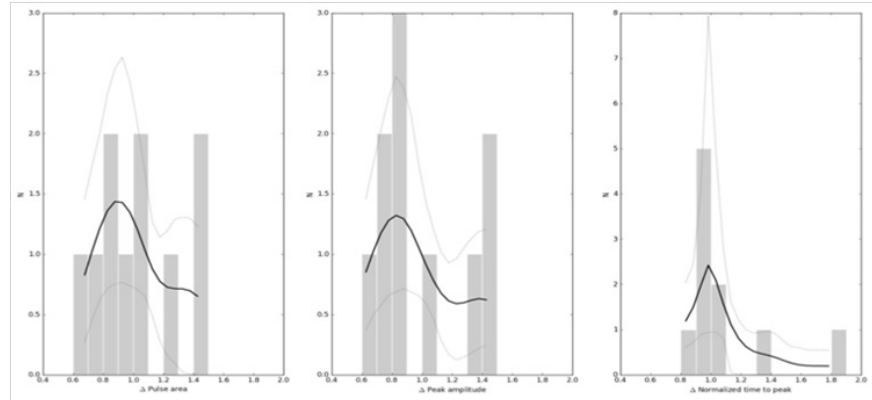

Figure 6 The CPI pulse, amplitude and time to normalize the pulse.

\section{Authors contribution}

Participated in the collect of samples: TARB. Conceived and designed the experiments: TARB, GF, DLSJ and SMO. Analyzed the data: DLSJ. Wrote the paper: TARB, DLSJ, GF.

\section{Acknowledgements}

None.

\section{Conflict of interest}

The authors declare that they have no competing interests.

\section{References}

1. Sevilla NL, Gardner JW. G-induced loss of consciousness: case-control study of 78 G-Locs in the F-15, F-16, and A-10. Aviat Space Environ Med. 2005;76(4):370-374

2. Wilson GF, Reis GA, Tripp LD. EEG correlates of G-induced loss of consciousness. Aviat Space Environ Med. 2005;76(1):19-27.

3. Maurino DE. Beyond aviation human factors: safety in high technology systems. UK: Ashgate Publishing Company; 1997. 181 p.

4. Galvagno SM, Massa TV, Price SC. Acceleration risk in student fighter pilots: preliminary analysis of a management program. Aviat Space Environ Med. 2004;75(12):1077-1080.

5. Hargens AR, Richardson S. Cardiovascular adaptations, fluid shifts, and countermeasures related to space flight. Respir Physiol Neurobiol. 2009;169(Suppl 1):S30-S33.

6. Gopalakrishnan R, Genc KO, Rice AJ, et al. Muscle volume, strength, endurance, and exercise loads during 6-month missions in space. Aviat Space Environ Med. 2010;81(2):91-102.

7. Schlegel TT, Wood SJ, Brown TE, et al. Effect of $30-\mathrm{min}+3 \mathrm{Gz}$ centrifugation on vestibular and autonomic cardiovascular function. Aviat Space Environ Med. 2003;74(7):717-724.

8. Chen HH, Wu YC, Kuo MD. An electromyographic assessment of the anti-G straining maneuver. Aviat Space Environ Med. 2004;75(2):162167.

9. Albery WB. Acceleration in other axes affects $+\mathrm{Gz}$ tolerance: dynamic centrifuge simulation of agile flight. Aviat Space Environ Med. 2004;75(1):1-6.

10. Hargens AR, Bhattacharya R, Schneider SM. Space physiology VI: exercise, artificial gravity, and countermeasure development for prolonged space flight. Eur J Appl Physiol. 2013;113(9):2183-2192.

11. Arbeille P, Provost R, Zuj K, et al. Measurements of jugular, portal, femoral, and calf vein cross-sectional area for the assessment of venous blood redistribution with long duration spaceflight (Vessel Imaging Experiment). Eur J Appl Physiol. 2015;115(10):2099-2106.

12. Macias BR, Liu JHK, Grande-Gutierrez N, et al. Intraocular and Intracranial Pressures During Head-Down Tilt with Lower Body Negative Pressure. Aviat Space Environ Med. 2015;86(1):3-7.

13. Jia H, Cui G, Xie S, et al. Vestibular function in military pilots before and after $10 \mathrm{~s}$ at $+9 \mathrm{Gz}$ on a centrifuge. Aviat Space Environ Med. 2009;80(1):20-23.

14. Mori E, Ishikawa M, Kato T, et al. Guidelines for management of idiopathic normal pressure hydrocephalus: second edition. Neurol Med Chir (Tokyo). 2012;52(11):775-809. 
15. Knudson R, McMillan D, Doucette D, et al. A comparative study of G-induced neck injury in pilots of the F/A-18, A-7, and A-4. Aviat Space Environ Med. 1988;59(8):758-760.

16. Barker PD. Reduced G tolerance associated with supplement use. Aviat Space Environ Med. 2011;82(2):140-143.

17. Watenpaugh DE, Smith ML. Human cardiovascular acclimation to microgravity. J Gravit Physiol. 1998;5(1):15-18.

18. Han WQ, Hu WD, Dong MQ, et al. Cerebral hemodynamics and brain functional activity during lower body negative pressure. Aviat Space Environ Med. 2009;80(8):698-702.

19. Oppenheim AV, Schafer RW, Buck JR. Discrete Time Signal Processing London: Pearson; 1999. 870 p.

20. Benitez D, Gaydecki PA, Zaidi A, et al. The use of the Hilbert transform in ECG signal analysis. Comput Biol Med. 2001;31(5):399-406.

21. Hastie T, Tibshirani R, Friedman J. The Elements of Statistical Learning. Elements. 2009;1:337-387.

22. Gibbons JD, Chakraborti S. Nonparametric Statistical Inference. New York: Marcel Dekker; 2003. 672 p.

23. Wiener TC. Space obstructive syndrome: Intracranial hypertension, intraocular pressure, and papilledema in space. Aviat Space Environ Med. 2012;83(1):64-66.

24. Yuh EL, Dillon WP. Intracranial hypotension and intracranial hypertension. Neuroimaging Clin N Am. 2010;20(4):597-617.

25. Alexander DJ, Gibson CR, Hamilton DR, et al. Risk of SpaceflightInduced Intracranial Hypertension and Vision Alterations. USA: Nasa; 2012. 109 p

26. Tripp LD, Warm JS, Matthews G, et al. $+\mathrm{Gz}$ acceleration loss of consciousness: Time course of performance deficits with repeated experience. Hum Factors. 2006;48(1):109-120.

27. Schrouff J, Perlbarg V, Boly M, et al. Brain functional integration decreases during propofol-induced loss of consciousness. Neuroimage. 2011;57(1):198-205.
28. Aponte VM, Finch DS, Klaus DM. Considerations for non-invasive in-flight monitoring of astronaut immune status with potential use of MEMS and NEMS devices. Life Sci. 2006;79(14):1317-1333.

29. Melek WW, Lu Z, Kapps A, et al. Modeling of dynamic cardiovascular responses during G-transition-induced orthostatic stress in pitch and roll rotations. IEEE Trans Biomed Eng. 2002;49(12 Pt 12):1481-1490.

30. Heldt T, Shim EB, Kamm RD, et al. Computational modeling of cardiovascular response to orthostatic stress. $J$ Appl Physiol. 2002;92(3):1239-1254.

31. Carpenter MG, Allum JH, Honegger F. Vestibular influences on human postural control in combinations of pitch and roll planes reveal differences in spatiotemporal processing. Exp Brain Res. 2001;140(1):95-111.

32. Michael AP, Marshall-Bowman K. Spaceflight-Induced Intracranial Hypertension. Aerosp Med Hum Perform. 2015;86(6):557-562.

33. Newman DG, Callister R. Cardiovascular training effects in fighter pilots induced by occupational high G exposure. Aviat Space Environ Med. 2008;79(8):774-778.

34. Stevenson AT, Scott JPR. +Gz Tolerance, with and without muscle tensing, following loss of anti-g trouser pressure. Aviat Space Environ Med. 2014;85(4):426-432.

35. Whinnery JE. $+\mathrm{G}(\mathrm{z})$ tolerance correlation with clinical parameters. Aviat Space Environ Med. 1979; p. 736-741.

36. Kikukawa A, Tachibana S, Yagura S. G-related musculoskeletal spine symptoms in Japan Air Self Defense Force F-15 pilots. Aviat Space Environ Med. 1995;66(3):269-272.

37. Steiner LA, Coles JP, Czosnyka M, et al. Cerebrovascular pressure reactivity is related to global cerebral oxygen metabolism after head injury. J Neurol Neurosurg Psychiatry. 2003;74(6):765-770.

38. Yates BJ, Kerman IA. Post-spaceflight orthostatic intolerance: Possible relationship to microgravity-induced plasticity in the vestibular system. Brain Res Rev. 1998;28(1-2):73-82. 